\title{
ON A CONNECTION BETWEEN POWERS OF OPERATORS AND FRACTIONAL CAUCHY PROBLEMS
}

\author{
VALENTIN KEYANTUO AND CARLOS LIZAMA
}

\begin{abstract}
Many phenomena in mathematical physics and in the theory of stochastic processes are recently described through fractional evolution equations. We investigate a general framework for connections between ordinary non homogeneous equations in Banach spaces and fractional Cauchy problems. When the underlying operator generates a strongly continuous semigroup, it is known, using a subordination argument, that the fractional evolution equation is well posed. In this case, we provide an explicit form of the solution involving special functions, one example being the Airy function.
\end{abstract}

\section{INTRODUCTION}

In the last years, many phenomena in physics and in the theory of stochastic processes lead with derivatives of non-integer order. In this paper, we are concerned with the connection between well- posedness of the following two evolution equations:

$$
\left\{\begin{array}{l}
v^{\prime}(t)=A^{n} v(t)+g(t), \quad t>0, \\
v(0)=x
\end{array}\right.
$$

and

$$
\begin{cases}D_{t}^{1 / n} u(t) & =A u(t), \quad t>0 \\ u(0) & =x\end{cases}
$$

Here, $A$ is a closed linear operator with domain $D(A)$ defined in some Banach space $X$ and $n \in \mathbb{N}$. The question of interest is: under what conditions on the operator $A$ and the $X$-valued function $g(\cdot)$ do the initial value $x$ problems (1.1) and (1.2) have the same unique solution?.

This close and somewhat unexpected relationship between equations (1.1) and (1.2) has appeared recently in the literature. For example, in fluids dynamics by Kulish and Lage [12] in case $A=\partial / \partial x$ and $n=2$ or, in case $A=\Delta$ and $n=2$ in the context of stochastic processes by Baeumer, Meerschaert and Nane [4]. Indeed, the connections of iterated Brownian motion and the bi-Laplacian have been found by Allouba and Zheng [1] and by DeBlassie [8]. Then Baeumer, Meerschaert and Nane [4] proved that when $A$ is the generator of a certain semigroup associated to a Markov process, then the initial value problems (1.1) and (1.2) have the same unique solution with $n=2$ and $n=3$ respectively (cf.[4, Theorems 3.6 and 3.9]).

Our main results show that the above connection between time and space for partial differential equations, observed in [4] and [12] (see also [1,2,8] and [15]), is valid when $A$ is the generator of an arbitrary $C_{0}$-semigroup defined on a Banach space $X$, and more generally when $A$ generates an

2000 Mathematics Subject Classification. 47D06, 35C05, 60J35, 26A33, 35K90, 34G10.

Key words and phrases. Perturbed fourth order PDEs, Cauchy problem, iterated Brownian motion, Caputo derivative, $C_{0}$-semigroups, PDE connection, $\alpha$-resolvent families, Wright function.

Both authors are partially financed by Laboratorio de Análisis Estocástico, Proyecto PBCT-ACT 13. The second author was also partially supported by VRID-DICYT, USACH. 
$\alpha$-resolvent family with $\alpha=\frac{1}{2}$ (see Definition 2.5). Note that, if $A$ is a nonnegative self-adjoint operator on a Hilbert space, then one cannot apply semigroup theory directly to solve problem (2.9). However, our results allows us to construct a solution.

In the case $n=2$ we are able to extend, and hence recover in Corollary 2.12 , the main result in $[4$, Theorem 3.1] (cf.also Theorem 0.1 in [1]) and, in passing, we generalize to the abstract setting the conditions that the initial data must satisfy in order that the equations to have a strong solution. This fact in the case of $A^{2}=\Delta^{2}$, the bi-Laplacian, has been analyzed and justified in [15, Lemma 3.1 and Lemma 3.3], after previous work of Allouba [2]. We show in this paper that the condition $f \in D(A)$ on the initial data is, in fact, a consequence of better regularity of the semigroup in case e.g. of $A=\Delta$, the Laplacian. The general result reads as follows.

Theorem 1.1. Assume that $A$ generates an analytic semigroup $T(t)$ on $X$. For $f \in D(A)$, the function

$$
u(t)=\frac{1}{\sqrt{\pi t}} \int_{0}^{\infty} e^{-s^{2} / 4 t} T(s) f d s, \quad t>0
$$

solves the problems

$$
\left\{\begin{array}{l}
v^{\prime}(t)=A^{2} v(t)+\frac{1}{\sqrt{\pi t}} A f, \quad t>0 \\
v(0)=f
\end{array}\right.
$$

and

$$
\left\{\begin{aligned}
D_{t}^{1 / 2} u(t) & =A u(t), \quad t>0 \\
u(0) & =f .
\end{aligned}\right.
$$

In addition, we will give an explicit formula for the solution of equations (1.1)-(1.2) in case $n=3$ which involves the Airy function instead of the Gaussian function in (1.3). Airy functions commonly appear in physics, especially in optics and quantum mechanics. Our approach allows one to solve for example the evolution equation involving the operator $A$ which can be an arbitrary variable coefficient second order differential operator with boundary conditions.

In particular, our analysis will show that the fractional Cauchy problem (1.2) shows a oscillatory behavior, in the temporal variable, between a dispersive and a diffusive equation, depending on the parity of $n$. This qualitative property of fractional partial differential equations seems to be new.

The paper is organized as follows: We consider the cases $n=2$ and $n=3$ in Section 2. There, we first use Laplace transform and then subordination arguments. The general case is presented in the last section. In our main general theorem, namely Theorem 3.3, we prove that when the operator $A$ generates a strongly continuous semigroup in $X$, then one obtains the solution of (1.2) by solving problem (1.1) with $g(t)=\sum_{k=1}^{n-1} \frac{t^{\frac{k}{n}-1}}{\Gamma\left(\frac{k}{n}\right)} A^{k} f$, where $f \in D\left(A^{n}\right)$ and $\Gamma(\cdot)$ is the Euler gamma function. Comparing the cases $n=2,3 \ldots$ we observe that a smaller fractional differentiation exponent increases the number of free constants in the ordinary differential equation. This phenomena is related with the lack of commutativity and the non-validity of the law of exponents for the fractional derivative.

In order to prove Theorem 3.3 we first consider in Proposition 3.1 an auxiliary ordinary differential equation related to the Wright functions, which can be of own interest. In Section 2, we consider the cases $n=2$ and $n=3$ while the general case is studied and stated in Section 3 .

$$
\text { 2. THE CASES } n=2 \text { AND } n=3
$$

There are various concepts of fractional derivatives connected with diverse areas of mathematics and the mathematical sciences. These notions are connected with several mathematicians, among 
them Liouville, Riemann, Weyl, Grünwald, Caputo and many others. In this paper, we deal with the Caputo fractional derivative, defined as

$$
D_{t}^{\alpha} u(t):=\frac{1}{\Gamma(1-\alpha)} \int_{0}^{t} \frac{u^{\prime}(\tau)}{(t-\tau)^{\alpha}} d \tau, \quad 0<\alpha<1,
$$

whenever $u \in C^{1}\left(\mathbb{R}_{+}, X\right)$. In (2.1), $\Gamma$ is the Euler gamma function. One of the main advantages of the Caputo fractional derivative is that initial conditions are expressed in terms of initial values of integer order derivatives. As a consequence, the Caputo fractional derivative appears more suitable to be treated by the Laplace transform technique in that it requires the knowledge of the initial values of the function and of its integer order derivatives. We have the following rule for the Laplace transform (see $[5, \mathrm{p} .12]$ and the references therein)

$$
\widehat{D_{t}^{\alpha}} u(\lambda)=\lambda^{\alpha} \hat{u}(\lambda)-\lambda^{\alpha-1} u(0), \quad \operatorname{Re} \lambda>0, \quad 0<\alpha<1 .
$$

Actually, the Caputo fractional $D_{t}^{\alpha}$ derivative can be defined for $\alpha$ in the range $(0, \infty)$. We will restrict our considerations to the case $\alpha \in(0,1)$.

The entire function

$$
\phi(\rho, \beta ; z)=\sum_{k=0}^{\infty} \frac{z^{k}}{k ! \Gamma(\rho k+\beta)}, \quad \rho>-1, z \in \mathbb{C},
$$

was introduced for the first time in the case $\rho>0$ by Wright in [19] in connection with his investigations in the asymptotic theory of partitions.

The important special case of the Wright function, namely, the functions $\Phi_{\alpha}(z):=\phi(-\alpha, 1-$ $\alpha,-z)$ in the case $0<\alpha<1$ has been considered in detail in [11] (where is denoted $M(z ; \alpha)$ and called the Mainardi function). For $\alpha=1 / m$ with an integer $m \geq 2$, these functions can be expressed as a sum of $(m-1)$ simpler entire functions as follows:

$$
\Phi_{1 / m}(t)=\frac{1}{\pi} \sum_{n=1}^{m-1} \Gamma\left(\frac{n}{m}\right) \sin \left(\frac{\pi n}{m}\right) F(t, n, m),
$$

with

$$
F(t, n, m)=\sum_{k=0}^{\infty}(-1)^{k(m+1)} \frac{\Gamma\left(\frac{n}{m}+k\right)}{\Gamma\left(\frac{n}{m}\right)} \frac{t^{m k+n-1}}{(m k+n-1) !} .
$$

Moreover, for $0<\gamma<1, \Phi_{\gamma}(t)$ is a probability density function, i.e. it satisfies

$$
\Phi_{\gamma}(t) \geq 0, t>0 ; \quad \int_{0}^{\infty} \Phi_{\gamma}(t) d t=1 .
$$

In the simplest cases $m=2$ and $m=3$ it was shown (see [11] and the references therein) that

$$
\begin{gathered}
\Phi_{1 / 2}(t) \equiv M(z ; 1 / 2)=\frac{1}{\sqrt{\pi}} e^{-z^{2} / 4} \\
\Phi_{1 / 3}(t) \equiv M(z ; 1 / 3)=3^{2 / 3} A i\left(\frac{z}{3^{1 / 3}}\right)
\end{gathered}
$$

where $A i(z)$ is the the Airy function.

We give the definition of the solution operator for the general class of equations to be considered in this paper. Let $A$ and $B$ be closed and linear operators in the Banach space $X$ and $0<\alpha<1$. 
We consider the equations:

$$
\begin{cases}D_{t}^{\alpha} u(t) & =A u(t), t>0 \\ u(0) & =x\end{cases}
$$

and

$$
\left\{\begin{array}{l}
v^{\prime}(t)=B v(t)+g(t), t>0 \\
v(0)=x .
\end{array}\right.
$$

Definition 2.1. (1) A function $u(\cdot)$ is a solution of (2.7) if $u(\cdot)$ is continuous on $[0, \infty)$, continuously differentiable on $(0, \infty)$ with $u(t) \in D(A)$ for all $t>0$ and (2.7) is satisfied.

(2) A function $v(\cdot)$ is a solution of (2.8) if $v(\cdot)$ is continuous on $[0, \infty)$, continuously differentiable on $(0, \infty)$ with $v(t) \in D(B)$ for all $t>0$ and (2.8) is satisfied.

The notion of exponentially bounded solution is defined in a standard way. In some cases we consider solutions obtained using subordination arguments. In these situations, the solution will actually be analytic.

Let $\Sigma_{\phi} \subset \mathbb{C}$ denote the open sector

$$
\Sigma_{\phi}=\{\lambda \in \mathbb{C} \backslash\{0\}:|\arg \lambda|<\phi\} .
$$

For a closed linear operator $B$ we denote by $\rho(B)$ and $\operatorname{ran}(\mathrm{B})$ the resolvent set and the range of $B$ respectively.

Theorem 2.2. Let $X$ be a Banach space and $A$ a closed linear operator on $X$. Suppose $\Sigma_{\pi / 2} \subset$ $\rho\left(A^{2}\right)$. For $f \in D(A)$, the problems

$$
\left\{\begin{aligned}
v^{\prime}(t) & =A^{2} v(t)+\frac{1}{\sqrt{\pi t}} A f, \quad t>0 \\
v(0) & =f
\end{aligned}\right.
$$

and

$$
\left\{\begin{aligned}
D_{t}^{1 / 2} u(t) & =A u(t), \quad t>0 \\
u(0) & =f
\end{aligned}\right.
$$

have both a same exponentially bounded solution.

Proof. Let $u$ be an exponentially bounded solution of (2.10), say, with $\|u(t)\| \leq M e^{\omega_{1} t}$ for all $t \geq 0$ where $\omega_{1} \geq 0$. Then the Laplace transform $\hat{u}(\lambda)$ exists for all $\operatorname{Re} \lambda>\omega_{1}$. Taking Laplace transform to $(2.10)$ we obtain using $(2.2)$

$$
\left(\lambda^{1 / 2}-A\right) \hat{u}(\lambda)=\lambda^{-1 / 2} f
$$

for all $\operatorname{Re} \lambda>\omega_{1}$. Since $f \in D(A)$ we can apply $\left(\lambda^{1 / 2}+A\right)$ to the above equation and obtain

$$
\left(\lambda-A^{2}\right) \hat{u}(\lambda)=\lambda^{-1 / 2}\left(\lambda^{1 / 2}+A\right) f=f+\lambda^{1 / 2} A f,
$$

for all $\operatorname{Re} \lambda>\omega_{1}$. Since $\Sigma_{\pi / 2} \subset \rho\left(A^{2}\right)$ we conclude that

$$
\hat{u}(\lambda)=\left(\lambda-A^{2}\right)^{-1}\left(I+\lambda^{1 / 2} A\right) f,
$$

for all $\operatorname{Re} \lambda>\omega_{1}$. Let $v$ be now an exponentially bounded solution of $(2.9)$, with $\|v(t)\| \leq M e^{\omega_{2} t}$ for all $t \geq 0$ where $\omega_{2} \geq 0$. Taking Laplace transform we get

$$
\left(\lambda-A^{2}\right) \hat{v}(\lambda)=f+\lambda^{1 / 2} A f, \quad \operatorname{Re} \lambda>\omega_{2} .
$$

It follows that $\hat{u}(\lambda)=\hat{v}(\lambda)$ for $\operatorname{Re} \lambda>\omega:=\max \left\{\omega_{1}, \omega_{2}\right\}$ and hence $u=v$ by uniqueness of the Laplace transform. This shows that if $u$ solves (2.10) then $u$ solves (2.9). 
Conversely, from (2.14) and since $\Sigma_{\pi / 2} \subset \rho\left(A^{2}\right)$ we obtain

$$
\begin{aligned}
\left(\lambda^{1 / 2}-A\right) \hat{v}(\lambda) & =\left(\lambda^{1 / 2}-A\right)\left(\lambda-A^{2}\right)^{-1}\left(I+\lambda^{1 / 2} A\right) f \\
& =\lambda^{1 / 2}\left(\lambda-A^{2}\right)^{-1} f+\left(\lambda-A^{2}\right)^{-1} A f \\
& -A\left(\lambda-A^{2}\right)^{-1} f-\lambda^{1 / 2} A\left(\lambda-A^{2}\right)^{-1} A f \\
& =\lambda^{1 / 2}\left(\lambda-A^{2}\right)^{-1} f-\lambda^{1 / 2} A^{2}\left(\lambda-A^{2}\right)^{-1} f
\end{aligned}
$$

where we have used the fact that $A$ commutes with $\left(\lambda-A^{2}\right)^{-1}$ on $D(A)$. Then, multiplying by $\lambda^{-1 / 2}$ the identity $I=\lambda\left(\lambda-A^{2}\right)^{-1}-A^{2}\left(\lambda-A^{2}\right)^{-1}$ we get that

$$
\left(\lambda^{1 / 2}-A\right) \hat{v}(\lambda)=\lambda^{-1 / 2} f
$$

for all Re $\lambda$ large enough. We conclude that if $v$ solves (2.9) then $v$ solves (2.10). This proves the theorem.

Example 2.3. (1) Suppose $B$ generates a strongly continuous group $(U(t))$ in $X$. Then $A=B^{2}$ generates a strongly continuous analytic semigroup (and in fact a cosine function) $(T(t))$. This semigroup is given through the abstract Weierstrass formula (see e.g. [3, Corollary 3.7.15]). Then (2.9) can be solved through the variation of constant formula:

$$
v(t)=T(t) f+\int_{0}^{t} T(t-s) A f \frac{d s}{\sqrt{\pi s}}, t>0 .
$$

(2) Take for $A$ a negative self adjoint operator on a Hilbert space $H$, so that $A$ generates a holomorphic semigroup of angle $\frac{\pi}{2}$. Then $A^{2}$ is not the generator of a strongly continuous semigroup on $H$ unless $A$ is bounded. Therefore, the solution to equation (2.9) cannot be obtained by semigroup arguments. Moreover, although the $H$-valued function $g(t)=$ $\frac{1}{\sqrt{\pi t}}$ Af belongs to $L_{l o c}^{1}(0, \infty ; H)$, it does not belong to $L_{l o c}^{p}(0, \infty ; H)$ if $p \geq 2$.

The assumption of Theorem 2.2 can be weakened considerably. This reformulation is important for the application to the concrete problems presented in Section 1. In particular, we will be able to apply the result to the case where $A$ is the generator of a strongly continuous semigroup on $X$ and more generally when $A$ generates an $\alpha$-resolvent family with $\alpha=\frac{1}{2}$. For instance, if $A$ is a nonnegative self-adjoint operator on a Hilbert space, then one cannot apply semigroup theory directly to solve problem (2.9). However, the result below allows us to construct a solution, the uniqueness of which is then guaranteed by the well-posedness of (2.10).

Theorem 2.4. Suppose $S=\rho\left(A^{2}\right) \cap \Sigma_{\pi / 2}$ has a limit point in $\Sigma_{\pi / 2}$. For $f \in D(A)$, the problems (2.9) and (2.10) have both the same exponentially bounded strong solution.

Proof. Without loss of generality, we may assume that the solutions $u$ and $v$ satisfy $\|u(t)\|+\|v(t)\| \leq$ $M e^{\omega t}, t \geq 0$ where $M \geq 1$ and $\omega \geq 0$. In fact, if $u$ is a solution of (2.9), then proceeding as in the preceding proof, we obtain

$$
\hat{u}(\lambda)=\left(\lambda-A^{2}\right)^{-1}\left(I+\lambda^{1 / 2} A\right) f,
$$

for all $\lambda \in S$. Similarly, we get that the solution $v$ of (2.9) satisfies

$$
\hat{v}(\lambda)=\left(\lambda-A^{2}\right)^{-1}\left(I+\lambda^{1 / 2} A\right) f
$$

for all $\lambda \in S$. Therefore, the two functions $\hat{u}(\lambda)$ and $\hat{v}(\lambda)$ which are analytic on $\{\lambda, \operatorname{Re} \lambda>\omega\}$, coincide for $\lambda \in S$. Since $S$ has a limit point in $\Sigma_{\pi / 2}$, it follows that the two functions must coincide for $\operatorname{Re} \lambda>\omega$. 
The converse assertion is handled in a similar manner.

Applying functional analytical methods the solvability of the problem (2.7), by means of the properties of solution operator families, was systematically investigated in [5]. This operator theoretical framework will be very important for our purposes.

Definition 2.5. Let $A$ be a closed linear operator with domain $D(A)$ defined on a Banach space $X$ and $\alpha>0$. We call $A$ the generator of an $\alpha$-resolvent family if there exists $\omega \geq 0$ and a strongly continuous function $S_{\alpha}: \mathbb{R}_{+} \rightarrow \mathcal{B}(X)$ such that $\left\{\lambda^{\alpha}: \operatorname{Re} \lambda>\omega\right\} \subset \rho(A)$ and

$$
\lambda^{\alpha-1}\left(\lambda^{\alpha}-A\right)^{-1} x=\int_{0}^{\infty} e^{-\lambda t} S_{\alpha}(t) x d t, \quad \operatorname{Re} \lambda>\omega, \quad x \in X .
$$

In this case, $S_{\alpha}(t)$ is called the $\alpha$-resolvent family generated by $A$.

Because of the uniqueness of the Laplace transform, a 1-resolvent family is the same as a $C_{0^{-}}$ semigroup whereas a 2-resolvent family corresponds to the concept of cosine family. The concept of $\alpha$-resolvent family was explicitly introduced by Bazhlekova [5]. It was already implicitly present in the paper [13] and in the monograph [18].

As in the situation of $C_{0}$-semigroups we have various relations between an $\alpha$-resolvent family and its generator. We denote

$$
g_{\beta}(t):=\frac{t^{\beta-1}}{\Gamma(\beta)}, \quad t>0, \quad \beta>0
$$

For convenience, we may set $g_{0}=\delta_{0}$, the Dirac measure concentrated at the origin. The following result is a direct consequence of [13, Proposition 3.1 and Lemma 2.2].

Proposition 2.6. Let $0<\alpha \leq 2$ and let $S_{\alpha}(t)$ be an $\alpha$-resolvent family on $X$ with generator $A$. Then the following holds:

(a) $S_{\alpha}(t) D(A) \subset D(A)$ and $A S_{\alpha}(t) x=S_{\alpha}(t) A x$ for all $x \in D(A), t \geq 0$;

(b) Let $x \in D(A)$ and $t \geq 0$. Then

$$
S_{\alpha}(t) x=x+\int_{0}^{t} g_{\alpha}(t-s) A S_{\alpha}(s) x d s
$$

(c) Let $x \in X$ and $t \geq 0$. Then $\int_{0}^{t} g_{\alpha}(t-s) S_{\alpha}(s) x d s \in D(A)$ and

$$
S_{\alpha}(t) x=x+A \int_{0}^{t} g_{\alpha}(t-s) S_{\alpha}(s) x d s
$$

In particular, $S_{\alpha}(0)=I$.

Assume that $A$ is the generator of a $1 / 2$-resolvent family $S_{1 / 2}(t)$. Then (2.11) shows that

$$
u(t)=S_{1 / 2}(t) f
$$

is the unique solution of (2.10) for $f \in D(A)$ (see also [5, p.20] or [18]), but not at first glance of (2.9) or by using e.g. Theorem 2.2, since the condition $\Sigma_{\pi / 2} \subset \rho\left(A^{2}\right)$ may not be satisfied. However, we are able to prove the following result. 
Theorem 2.7. Assume that $A$ generates a $1 / 2$ - resolvent family $S_{1 / 2}(t)$ on $X$. For $f \in D\left(A^{2}\right)$, the function

$$
u(t)=S_{1 / 2}(t) f, \quad t>0
$$

solves the problems (2.9) and (2.10).

Proof. We know that for all $x \in D(A)$, the function $u(t)=S_{1 / 2}(t) x$ solves (2.9), and that is satisfies

$$
D_{t}^{1 / 2} S_{1 / 2}(t) x=\int_{0}^{t} g_{1 / 2}(s) S_{1 / 2}^{\prime}(t-s) x d s=A S_{1 / 2}(t) x, \quad t>0
$$

for all $x \in D(A)$. Hence, for $f \in D\left(A^{2}\right)$ we have $A S_{1 / 2}(t) f=S_{1 / 2}(t) A f \in D(A)$ and

$$
A^{2} S_{1 / 2}(t) f=A S_{1 / 2}(t) A f \text {. }
$$

Using (2.19) with $x=A f$ we obtain

$$
A^{2} S_{1 / 2}(t) f=D_{t}^{1 / 2} S_{1 / 2}(t) A f .
$$

On the other hand, by Proposition 2.6,

$$
S_{1 / 2}(t) f=f+\int_{0}^{t} g_{1 / 2}(s) S_{1 / 2}(t-s) A f d s,
$$

and hence

$$
\begin{aligned}
D_{t}^{1 / 2} S(t) & =S_{1 / 2}^{\prime}(t) f \\
& =g_{1 / 2}(t) S_{1 / 2}(0) A f+\int_{0}^{t} g_{1 / 2}(s) S_{1 / 2}^{\prime}(t-s) A f d s \\
& =g_{1 / 2}(t) A f+\int_{0}^{t} g_{1 / 2}(s) S_{1 / 2}^{\prime}(t-s) A f d s \\
& =\frac{1}{\sqrt{\pi t}} A f+A^{2} S_{1 / 2}(t) f
\end{aligned}
$$

where in the last equality we have used (2.42). The proof is complete.

Remark 2.8.

1) Examples of operators generating $1 / 2$-resolvent families but not generating a $C_{0}$-semigroup are given in [5].

2) Observe that we cannot proceed beyond the case $n=2$ with the above argument, because $S_{\alpha}(t)$ with $\alpha \in(0,1)$ is not differentiable at $t=0^{+}$, and hence the proof in case $n \geq 3$ breaks down in the analog part of the step (2.23).

3) We note that, in contrast with the case of equation (2.10), in general the solutions of the equation (2.9) are not necessarily unique. An example is given in [4].

In order to obtain an explicit representation of the solution, we note that by the subordination principle for $\alpha$-resolvent families ([5, Theorem 3.1] or [18, Theorem 4.2 and Corollary 4.5]), we have: If $A$ is the generator of a $\beta$-resolvent family $S_{\beta}(t)$, then $A$ is the generator of a $\alpha$-resolvent family $S_{\alpha}(t)$ for all $0<\alpha<\beta \leq 2$ and the following representation holds

$$
S_{\alpha}(t) x=\int_{0}^{\infty} t^{-\alpha / \beta} \Phi_{\alpha / \beta}\left(s t^{-\alpha / \beta}\right) S_{\beta}(s) x d s, \quad x \in X, \quad t>0 .
$$

In particular, if $A$ is the generator of a $C_{0}$-semigroup $T(t)$, we have

$$
S_{1 / 2}(t) x=\int_{0}^{\infty} t^{-1 / 2} \Phi_{1 / 2}\left(s t^{-1 / 2}\right) T(s) x d s, \quad x \in X, \quad t>0 .
$$


Recall that $\Phi_{1 / 2}\left(s t^{-1 / 2}\right)=\frac{1}{\sqrt{\pi}} e^{-s^{2} / 4 t}$. We conclude that

$$
S_{1 / 2}(t) x=\frac{1}{\sqrt{\pi t}} \int_{0}^{\infty} e^{-s^{2} / 4 t} T(s) x d s, \quad x \in X, t>0 .
$$

The previous observations give an immediate consequence of the above theorem that we state in the following result.

Theorem 2.9. Assume that $A$ generates a $C_{0}$-semigroup $T(t)$ on $X$. For $f \in D\left(A^{2}\right)$, the function

$$
u(t)=\frac{1}{\sqrt{\pi t}} \int_{0}^{\infty} e^{-s^{2} / 4 t} T(s) f d s, \quad t>0 .
$$

solves the problems (2.9) and (2.10).

Proof. We give an alternative proof. Is clear that (2.27) solves (2.10). It remains to show that the function $u(\cdot)$ solves $(2.9)$. Define $q(t, s)=\frac{1}{\sqrt{\pi t}} e^{-s^{2} / 4 t}$ and note that $\frac{\partial}{\partial t} q(t, s)=\frac{\partial^{2}}{\partial s^{2}} q(t, s)$. Then we can proceed as in [1] (see also [4, Remark 3.5]): Using integration by parts we obtain

$$
\begin{aligned}
\frac{\partial}{\partial t} S_{1 / 2}(t) f & =\int_{0}^{\infty} \frac{\partial}{\partial t} q(t, s) T(s) f d s \\
& =\int_{0}^{\infty} \frac{\partial^{2}}{\partial s^{2}} q(t, s) T(s) f d s \\
& =\left.q(t, s) \frac{\partial}{\partial s}[T(s) f]\right|_{s=0}+\int_{0}^{\infty} \frac{\partial^{2}}{\partial s^{2}}[T(s) f] q(t, s) d s
\end{aligned}
$$

Since $f \in D\left(A^{2}\right)$ we have $\frac{\partial^{2}}{\partial s^{2}} T(s) f=A^{2} T(s) f$ and therefore

$$
\begin{aligned}
\frac{\partial}{\partial t} S_{1 / 2}(t) f & =q(t, 0) A f+\int_{0}^{\infty} A^{2}[T(s) f] q(t, s) d s \\
& =\frac{1}{\sqrt{\pi t}} A f+A^{2} S_{1 / 2}(t) f,
\end{aligned}
$$

where the last step is justified because the operator $A$ is closed.

Remark 2.10. Note that the Riemann Liouville fractional derivative has the property that $\frac{\partial^{1 / 2} \mathbf{1}}{\partial t^{1 / 2}}=$ $\frac{1}{\sqrt{\pi t}}$, so that in certain sense the fractional derivative is present in the formula of (2.27).

Remark 2.11. Let $L_{x}$ be the generator of a continuous Markov semigroup $T(t) f(x)=E_{x}\left[f\left(X_{t}\right)\right]$ and take $f \in D\left(L_{x}^{2}\right)$, the domain of the generator, then we recover [4, Theorem 3.1] (see also [15, Theorem 2.1]). However, note that in [4] the condition $f \in D\left(L_{x}\right)$ instead of $f \in D\left(L_{x}^{2}\right)$ is assumed. This fact in the case of the bi-Laplacian has been analyzed and justified in [15, Lemma 3.1 and Lemma 3.3], after previous work of Allouba [2]. We remark here that such situation is, in fact, a consequence of better regularity of the semigroup in case e.g. of the Laplacian. We clarify the situation in what follows.

We note that if $A$ generates a strongly continuous semigroup $T(t)$ such that

$$
\operatorname{ran}(T(t)) \subset D(A) \text { for all } t>0
$$

then the map $t \rightarrow T(t) f \in X$ is infinitely often differentiable for all $t>0$ and $f \in X$ and we have (cf. [9, p.104]):

$$
T(t) f \in D\left(A^{k}\right)
$$


and

$$
A^{k} T(t) f=\frac{d^{k}}{d t^{k}} T(t) f
$$

for all $f \in X, k \in \mathbb{N}$ and $t>0$. Hence, we obtain the following important result, corresponding to Theorem 1.1 given in the introduction.

Corollary 2.12. Assume that $A$ generates an analytic semigroup $T(t)$ on a Banach space $X$. For $f \in D(A)$, the function in (2.27) solves the problems (2.9) and (2.10).

Proof. By [5, Theorem 3.3] the solution operator $S_{1 / 2}(t)$, obtained by subordination of the semigroup $T(t)$ and given in $(2.26)$, admits an analytic extension to the sector $\Sigma_{\pi / 2}$. Hence by [5, Proposition 2.15], $S_{1 / 2}(t)$ enjoys the following regularity

$$
S_{1 / 2}(t) x \in D(A) \text { for all } x \in X .
$$

As a consequence we have by Proposition 2.6,

$$
A S_{1 / 2}(t) f=S_{1 / 2}(t) A f \in D(A) \text { for } f \in D(A) .
$$

Hence, for $f \in D(A)$

$$
A^{2} S_{1 / 2}(t) f=A S_{1 / 2}(t) A f=A \int_{0}^{\infty}[T(s) A f] q(t, s) d s .
$$

Note that for an analytic semigroup on a Banach space $X$ one always has that $\operatorname{ran}(T(t)) \subset$ $D(A), t>0$ (see e.g. [9, Theorem 4.6]) implying that (2.29) and (2.30) holds. In particular, $T(s) A f \in D(A), s>0$ and since $A$ is closed, we obtain

$$
A^{2} S_{1 / 2}(t) f=\int_{0}^{\infty} A[T(s) A f] q(t, s) d s, t>0 .
$$

Finally, since $A T(s) A f=A^{2} T(s) f$ we obtain the assertion from (2.34), using (2.30) and following the alternative proof of Theorem 2.9 .

Remark 2.13. We note that we cannot go further the case $n=2$ with the argument of the proof of Corollary 2.12 because, in contrast to analytic semigroups, we cannot proceed beyond the regularity (2.31) in case of $\alpha$-resolvent families. This lack of regularity has been observed in [5] and proved in [18, Theorem 2.2(iii)] in the context of evolutionary integral Volterra equations.

Example 2.14. Consider in $X=L^{p}\left(\mathbb{R}^{N}\right), 1 \leq p<\infty$ the diffusion semigroup:

$$
T(t) f(x)=(4 \pi t)^{N / 2} \int_{\mathbb{R}^{N}} e^{-|x-s|^{2} / 4 t} f(s) d s, \quad t>0, x \in \mathbb{R}^{N}, f \in X .
$$

By putting $\mu_{t}(x)=(4 \pi t)^{-N / 2} e^{-|x|^{2} / 4 t}$, this can be written as

$$
T(t) f(x)=\mu_{t} * f(x)
$$

It is well known that $T(t)$ is a bounded analytic semigroup with generator $\Delta$. Hence, for $f \in D(\Delta)$ the function

$$
u(t, x)=\frac{1}{\sqrt{\pi t}} \int_{0}^{\infty} e^{-s^{2} / 4 t} T(s) f(x) d s, \quad t>0 .
$$

solves simultaneously the partial differential equation

$$
\left\{\begin{aligned}
\frac{\partial}{\partial t} v(t, x) & =\Delta^{2} v(t, x)+\frac{1}{\sqrt{\pi t}} \Delta f(x), \quad t>0 \\
v(0, x) & =f(x)
\end{aligned}\right.
$$


and the fractional partial differential equation

$$
\left\{\begin{aligned}
\frac{\partial^{1 / 2}}{\partial t^{1 / 2}} u(t, x) & =\Delta u(t, x), \quad t>0 \\
u(0, x) & =f(x)
\end{aligned}\right.
$$

Example 2.15. Suppose that $X(t)=x+X_{0}(t)$ where $X_{0}(t)$ is a Lévy process starting at zero. If $L_{x}$ is the generator in [4, (2.8)] of the semigroup $T(t) f(x)=E_{x}\left[f\left(X_{t}\right)\right]$ on $L^{1}\left(\mathbb{R}^{d}\right)$, then $L_{x}$ is the generator of a bounded analytic semigroup, because $T(t)$ is obtained by subordination of another semigroup (compare [4, formula (2.7)] and [6, formula (2)]). Hence, by Corollary 2.12, for any $f \in D\left(L_{x}\right)$ we recover [4, Theorem 3.6].

Example 2.16. In fluids dynamics, the momentum equation for a one-dimensional time-dependent viscous-diffusion problem of a semi-infinite fluid bounded by a flat plate, is

$$
\frac{\partial F(x, t)}{\partial t}=v \frac{\partial^{2} F(x, t)}{\partial x^{2}}
$$

(see Kulish and Lage [12]). Here one assumes constant and uniform viscosity and neglects convective media (advection). The function $F(x, t)$ is the fluid vorticity, or the fluid velocity in case of negligible pressure effect, $t$ is the time, $v$ is the fluid kinematic viscosity and $x$ is the spacial coordinate normal to, and with origin at, the plate. It is also assumed that the fluid is initially at equilibrium, so that $F(x, t<0)=F_{0}$, with $F_{0}$ being a constant value. Using the properties of the fractional calculus, in [12] it was observed that equation (2.40) can be rewritten as

$$
v^{-1 / 2} \frac{\partial^{1 / 2} F(x, t)}{\partial t^{1 / 2}}=(\pi v t)^{-1 / 2} F_{0}-\frac{\partial F(x, t)}{\partial x},
$$

where $\frac{\partial^{1 / 2}}{\partial t^{1 / 2}}$ indicates the Riemann-Liouville half derivative. Therefore, the viscous-diffusion equation (2.40), which is a classical partial differential equation of first order in time and second order in space, is transformed into a partial differential equation of fractional order order in time and first order in the space variable. This transformation is general and not restricted by any additional assumptions on the physics of the process in question.

We observe that (2.40) and (2.41) can be written as

$$
\frac{\partial F(x, t)}{\partial t}=A_{x}^{2} F(x, t)
$$

and

$$
\frac{\partial^{1 / 2} F(x, t)}{\partial t^{1 / 2}}=(\pi t)^{-1 / 2} F_{0}+A_{x} F(x, t) .
$$

respectively, where $A_{x}:=-v^{1 / 2} \frac{\partial}{\partial x}$ is the generator of a translation $C_{0}$-semigroup. As remarked, $\frac{\partial^{1 / 2}}{\partial t^{1 / 2}}$ is the Riemann-Liouville fractional derivative instead of the Caputo fractional derivative considered in this paper. However, both fractional derivatives of a function $g$ are in general related by the formula:

$$
\frac{\partial^{1 / 2}}{\partial t^{1 / 2}}(g(t)-g(0))=D_{t}^{1 / 2} g(t),
$$

or, since $\frac{\partial^{1 / 2}}{\partial t^{1 / 2}} 1=\frac{1}{\sqrt{\pi t}}$, we obtain in our case

$$
\frac{\partial^{1 / 2} F(x, t)}{\partial t^{1 / 2}}-F(x, 0) \frac{1}{\sqrt{\pi t}}=D_{t}^{1 / 2} F(x, t),
$$

where $F(x, 0)=F_{0}$. Hence (2.43) is equivalent, in terms of the Caputo fractional derivative to

$$
D_{t}^{1 / 2} F(x, t)=A_{x} F(x, t)
$$


with $F(x, 0)=F_{0}$. In particular it shows that $A_{x} F(x, 0)=0$. Consequently, (2.42) is equivalent to

$$
\frac{\partial F(x, t)}{\partial t}=A_{x}^{2} F(x, t)+\frac{1}{\sqrt{\pi t}} A_{x} F(x, 0) .
$$

Therefore Theorem 2.9 applies in this case, recovering the results in [12].

Concerning the case $n=3$, we prove:

Theorem 2.17. Assume that $A$ generates a $C_{0}$-semigroup $T(t)$ on $X$. For $f \in D\left(A^{3}\right)$, the function

$$
u(t)=\frac{3}{\sqrt[3]{3 t}} \int_{0}^{\infty} A i(s / \sqrt[3]{3 t}) T(s) f d s, \quad t>0
$$

where Ai(x) denotes the Airy function, solves the problems

$$
\left\{\begin{array}{l}
u^{\prime}(t)=A^{3} u(t)+\frac{t^{-2 / 3}}{\Gamma(1 / 3)} A f+\frac{t^{-1 / 3}}{\Gamma(2 / 3)} A^{2} f \\
u(0)=f
\end{array}\right.
$$

and

$$
\left\{\begin{aligned}
D_{t}^{1 / 3} u(t) & =A u(t) \\
u(0) & =f
\end{aligned}\right.
$$

Proof. Since $A$ is the generator of a $C_{0}$-semigroup, by the subordination principle $A$ is the generator of a $1 / 3$-resolvent family given by

$$
S_{1 / 3}(t) f=\int_{0}^{\infty} t^{-1 / 3} \Phi_{1 / 3}\left(s t^{-1 / 3}\right) T(s) f d s, \quad f \in X
$$

and is clear that $u(t)=S_{1 / 3}(t) f$ solves (2.48). We know that $\Phi_{1 / 3}(t)=\phi(-1 / 3,2 / 3,-t)=$ $3^{2 / 3} A i\left(t / 3^{1 / 3}\right)$ (see [11, p.10]). In particular, $\Phi_{1 / 3}^{\prime \prime}(t)=\frac{1}{3} t \Phi_{1 / 3}(t)$. Denote $p(s, t)=t^{-1 / 3} \Phi_{1 / 3}\left(s t^{-1 / 3}\right)$.

Using the fact that the Airy function satisfies the differential equation $y^{\prime \prime}(z)-z y(z)=0$, we deduce that

$$
\frac{\partial p(s, t)}{\partial t}=-\frac{\partial^{3} p(s, t)}{\partial s^{3}}
$$

We note also that $p(0, t)=\frac{t^{-1 / 3}}{\Gamma(2 / 3)}, \frac{\partial p(0, t)}{\partial s}=-\frac{t^{-2 / 3}}{\Gamma(1 / 3)}$ and $\frac{\partial^{2} p(0, t)}{\partial s^{2}}=0$. This, together with the fact that $e^{\omega s} p(s, t) \rightarrow 0, e^{\omega s} \frac{\partial p(s, t)}{\partial s} \rightarrow 0$ and $e^{\omega s} \frac{\partial^{2} p(s, t)}{\partial s^{2}} \rightarrow 0$ as $s \rightarrow \infty$ for every $\omega \in \mathbb{R}(\mathrm{cf}$. [16, 
p.288-289] or [19]) and using integration by parts, we obtain

$$
\begin{aligned}
\frac{\partial}{\partial t} S_{1 / 3}(t) f & =\int_{0}^{\infty} \frac{\partial}{\partial t} p(s, t) T(s) f d s \\
& =-\int_{0}^{\infty} \frac{\partial^{3}}{\partial s^{3}} p(s, t) T(s) f d s \\
& =-\left.\frac{\partial^{2}}{\partial s^{2}} p(s, t) T(s) f\right|_{s=0} ^{\infty}+\left.\frac{\partial}{\partial s} p(s, t) A T(s) f\right|_{s=0} ^{\infty}-\left.p(s, t) A^{2} T(s) f\right|_{s=0} ^{\infty} \\
& +\int_{0}^{\infty} \frac{\partial^{3}}{\partial s^{3}}[T(s) f] p(s, t) d s \\
& =-\frac{\partial^{2}}{\partial s^{2}} p(0, t) A f-\frac{\partial}{\partial s} p(0, t) A f+p(0, t) A^{2} f+\int_{0}^{\infty} A^{3}[T(s) f] p(s, t) d s \\
& =\frac{t^{-2 / 3}}{\Gamma(1 / 3)} A f+\frac{t^{-1 / 3}}{\Gamma(2 / 3)} A^{2} f+A^{3} S_{1 / 3}(t) f,
\end{aligned}
$$

Remark 2.18. 1. Note that for the Riemann-Liouville fractional derivative we have $\frac{\partial^{1 / 3} 1}{\partial t^{1 / 3}}=\frac{t^{-1 / 3}}{\Gamma(2 / 3)}$ and $\frac{\partial^{2 / 3} \mathbf{1}}{\partial t^{2 / 3}}=\frac{t^{-2 / 3}}{\Gamma(1 / 3)}$.

2. As remarked in the introduction, comparing the cases $n=2$ and $n=3$ we observe that a smaller fractional differentiation exponent increases the number of free constants in the ordinary differential equation. This phenomena is related with the lack of commutativity and the non-validity of the law of exponents for the fractional derivative, i.e. in general $D_{t}^{\alpha} D_{t}^{\beta} \neq D_{t}^{\beta} D_{t}^{\alpha}$.

Example 2.19. Suppose that $X(t)=x+X_{0}(t)$ where $X_{0}(t)$ is a Lévy process starting at zero. If $L_{x}$ is the generator in $[4,(2.8)]$ of the semigroup $T(t) f(x)=E_{x}\left[f\left(X_{t}\right)\right]$ on $L^{1}\left(\mathbb{R}^{d}\right)$ then, for any $f \in D\left(L_{x}^{3}\right)$, we recover $[4$, Theorem 3.9].

We observe that in [4] no explicit formula, like (2.46) in terms of the Airy function, was given.

\section{The General CASE}

In this section we clarify the role played by the different actors in the previous results. In order to do this, the following result is fundamental.

Proposition 3.1. For all $m=2,3, \ldots$, the ordinary differential equation

$$
f^{(m-1)}(t)=(-1)^{m+1} t f(t),
$$

with initial conditions

$$
f^{(k-1)}(0)=\frac{(-\sqrt[m]{m})^{k-1}}{\Gamma\left(1-\frac{k}{m}\right)}, \quad k=1,2, \ldots, m-1
$$

has the unique solution

$$
f(t)=\Phi_{1 / m}(t \sqrt[m]{m})
$$

Proof. Let $m \geq 2$ be fixed. By simplicity of the notation, we define

$$
y(t):=\Phi_{1 / m}(t)=\phi\left(\frac{-1}{m}, 1-\frac{1}{m} ;-t\right)=\sum_{k=0}^{\infty} \frac{(-t)^{k}}{k ! \Gamma\left(1-\frac{k+1}{m}\right)},
$$


then a direct calculation, and also using the fact that $\frac{1}{\Gamma(0)}=0$, gives

$$
y^{(k-1)}(0)=\frac{(-1)^{k-1}}{\Gamma\left(1-\frac{k}{m}\right)}, k=1,2 \ldots m-1, \quad y^{(m-1)}(0)=0 .
$$

Then we obtain

$$
\begin{aligned}
\lambda^{m-1} y(0)+\lambda^{m-2} y^{\prime}(0)+\ldots+y^{(m-1)}(0) & =\sum_{k=1}^{m} \frac{(-1)^{k-1} \lambda^{m-k}}{\Gamma\left(1-\frac{k}{m}\right)} \\
& =\sum_{p=0}^{m-1} \frac{(-1)^{m-p-1} \lambda^{p}}{\Gamma\left(\frac{p}{m}\right)} \\
& =(-1)^{m-1} \sum_{k=1}^{m-1} \frac{(-1)^{k} \lambda^{k}}{\Gamma\left(\frac{k}{m}\right)}
\end{aligned}
$$

and, since $\Gamma\left(\frac{k}{m}+1\right)=\frac{k}{m} \Gamma\left(\frac{k}{m}\right)$, we get

$$
\begin{aligned}
\lambda^{m} \hat{y}(\lambda)-\lambda^{m-1} y(0)-\lambda^{m-2} y^{\prime}(0)-\ldots-y^{(m-1)}(0) & =\lambda^{m} \hat{y}(\lambda)+(-1)^{m} \sum_{k=1}^{m-1} \frac{(-1)^{k} \lambda^{k}}{\Gamma\left(\frac{k}{m}\right)} \\
& =\lambda^{m} \hat{y}(\lambda)+\frac{(-1)^{m}}{m} \sum_{k=1}^{m-1} \frac{k(-\lambda)^{k}}{\Gamma\left(1+\frac{k}{m}\right)} .
\end{aligned}
$$

On the other hand, we note that

$$
\hat{y}(\lambda)=E_{1 / m}(-\lambda)=\sum_{k=0}^{\infty} \frac{(-\lambda)^{k}}{\Gamma\left(1+\frac{k}{m}\right)}
$$

is the Mittag-Leffler function (see e.g. [5]). Then,

$$
\lambda[\hat{y}(\lambda)]^{\prime}=\sum_{k=1}^{\infty} \frac{k(-\lambda)^{k}}{\Gamma\left(1+\frac{k}{m}\right)}
$$

and

$$
\begin{aligned}
\lambda^{m} \hat{y}(\lambda) & =(-1)^{m} \sum_{n=0}^{\infty} \frac{(-\lambda)^{n+m}}{\Gamma\left(1+\frac{n}{m}\right)} \\
& =(-1)^{m} \sum_{k=m}^{\infty} \frac{(-\lambda)^{k}}{\Gamma\left(\frac{k}{m}\right)} \\
& =\frac{(-1)^{m}}{m} \sum_{k=m}^{\infty} \frac{k(-\lambda)^{k}}{\Gamma\left(1+\frac{k}{m}\right)}
\end{aligned}
$$

Hence, combining with (3.5), we obtain

$$
\begin{aligned}
\widehat{y^{(m)}}(\lambda) & =\frac{(-1)^{m}}{m} \sum_{k=1}^{\infty} \frac{k(-\lambda)^{k}}{\Gamma\left(1+\frac{k}{m}\right)} \\
& =\frac{(-1)^{m}}{m} \lambda[\hat{y}(\lambda)]^{\prime} .
\end{aligned}
$$

From the above, by uniqueness of the Laplace transform, and taking into account the fact that $y^{(m-1)}(0)=0$, we get that $y(t)$ satisfy the equation

$$
y^{(m-1)}(t)=\frac{(-1)^{m+1}}{m} t y(t) .
$$


Now, define

$$
f(t)=y(t \sqrt[m]{m})
$$

Then $f(t)$ satisfies

$$
f^{(m-1)}(t)=\frac{m}{\sqrt[m]{m}} y^{(m-1)}(t \sqrt[m]{m})=\frac{(-1)^{m+1}}{\sqrt[m]{m}} t \sqrt[m]{m} y(t \sqrt[m]{m})=(-1)^{m+1} t f(t)
$$

and

$$
f^{(k-1)}(0)=\frac{k}{\sqrt[k]{k}} y^{(k-1)}(0)=\frac{(-\sqrt[m]{m})^{k-1}}{\Gamma\left(1-\frac{k}{m}\right)} \quad k=1,2, \ldots, m-1
$$

Remark 3.2. In case $m=2$ the equation

$$
f^{\prime}(t)=t f(t)
$$

with initial condition $f(0)=\frac{1}{\Gamma(1 / 2)}=\frac{1}{\sqrt{\pi}}$ has the solution $f(t)=\frac{1}{\sqrt{2}} e^{-t^{2} / 2}$ which, in this case, can be calculated directly. In particular, we get $\Phi_{1 / 2}(t)=f(t / \sqrt{2})=\frac{1}{\sqrt{\pi}} e^{-t^{2} / 4}$ as established in the previous section.

In case $m=3$ the equation is

$$
f^{\prime \prime}(t)-t f(t)=0
$$

Equation (3.9) is known as the Airy equation or the Stokes equation. This is the simplest secondorder linear differential equation with a turning point (a point where the character of the solutions changes from oscillatory to exponential). With the initial conditions $f(0)=\frac{1}{\Gamma(2 / 3)}$ and $f^{\prime}(0)=$ $-\frac{\sqrt[3]{3}}{\Gamma(1 / 3)}$, equation (3.9) has the solution $f(t)=3^{2 / 3} A i(t)=\Phi_{1 / 3}(t \sqrt[3]{3})$, where Ai( $\left.x\right)$ denotes the Airy function.

The general case appears to be studied only very recently in the literature (cf. [10] where our equations (3.1), except by the term $(-1)^{m+1}$, is called a higher order Airy equation). As proved in [10], equations (3.1) are linked, by the Cole-Hopf transformation, to certain nonlinear differential equations that, in case of the Airy function, corresponds to a equation of Riccati type.

Our main result is the following.

Theorem 3.3. Let $m \geq 2$ be fixed. Assume that $A$ generates a $C_{0}$-semigroup $T(t)$ on $X$. For $f \in D\left(A^{m}\right)$, the function

$$
u(t)=\frac{1}{\sqrt[m]{t}} \int_{0}^{\infty} \Phi_{1 / m}(s / \sqrt[m]{t}) T(s) f d s, \quad t>0
$$

solves the problems

$$
\left\{\begin{aligned}
D_{t}^{1 / m} u(t) & =A u(t) \\
u(0) & =f
\end{aligned}\right.
$$

and

$$
\left\{\begin{array}{l}
u^{\prime}(t)=A^{m} u(t)+\sum_{k=1}^{m-1} \frac{t^{\frac{k}{m}-1}}{\Gamma\left(\frac{k}{m}\right)} A^{k} f \\
u(0)=f .
\end{array}\right.
$$

Proof. Define

$$
p(s, t)=t^{-1 / m} \Phi_{1 / m}\left(s t^{-1 / m}\right) .
$$


Since $A$ is the generator of a $C_{0}$-semigroup, by the subordination principle $A$ is the generator of a $1 / m$-resolvent family given by

$$
S_{1 / m}(t) f=\int_{0}^{\infty} p(s, t) T(s) f d s, \quad f \in X
$$

and is clear that $u(t)=S_{1 / m}(t) f$ solves (3.11). To prove that solves (3.12) we observe that

$$
\frac{\partial^{k}}{\partial s^{k}} p(s, t)=t^{-\frac{k+1}{m}} \Phi_{1 / m}^{(k)}\left(s t^{-1 / m}\right), \quad k=1,2, \ldots, m
$$

and

$$
(-1) \frac{\partial}{\partial t} p(s, t)=\frac{t^{-\frac{m+1}{m}}}{m}\left[\Phi_{1 / m}\left(s t^{-1 / m}\right)+s t^{-1 / m} \Phi_{1 / m}^{\prime}\left(s t^{-1 / m}\right)\right] .
$$

By Proposition 3.1 we have $\Phi_{1 / m}(v)=f(v / \sqrt[m]{m})$ where $f$ is the solution of equation (3.1). In consequence, we have

$$
\begin{aligned}
\Phi_{1 / m}^{(m)}(v) & =\frac{1}{m} f^{(m)}(v / \sqrt[m]{m})=\frac{(-1)^{m+1}}{m}\left[f(v / \sqrt[m]{m})+\frac{v}{\sqrt[m]{m}} f^{\prime}(v / \sqrt[m]{m})\right] \\
& =\frac{(-1)^{m+1}}{m}\left[\Phi_{1 / m}(v)+v \Phi_{1 / m}^{\prime}(v)\right] .
\end{aligned}
$$

We conclude that

$$
\frac{\partial^{m}}{\partial s^{m}} p(s, t)=(-1)^{m+1}(-1) \frac{\partial}{\partial t} p(s, t)=(-1)^{m} \frac{\partial}{\partial t} p(s, t) .
$$

Using the above relation in (3.14) and integration by parts, we obtain

$$
\begin{aligned}
u^{\prime}(t) & =\frac{\partial}{\partial t} S_{1 / m}(t) f=\int_{0}^{\infty} \frac{\partial}{\partial t} p(s, t) T(s) f d s \\
& =(-1)^{m} \int_{0}^{\infty} \frac{\partial^{m}}{\partial s^{m}} p(s, t) T(s) f d s \\
& =\left.\sum_{k=1}^{m}(-1)^{m+k-1} \frac{\partial^{m-k}}{\partial s^{m-k}} p(s, t) T^{(k-1)}(s) f\right|_{s=0} ^{s=\infty} \\
& +\int_{0}^{\infty} p(s, t) \frac{\partial^{m}}{\partial s^{m}} T(s) f d s .
\end{aligned}
$$

By (3.15) and (3.4) we have

and

$$
\frac{\partial^{k-1}}{\partial s^{k-1}} p(0, t)=t^{-k / m} \Phi_{1 / m}^{(k-1)}(0)=t^{-k / m} \frac{(-1)^{k-1}}{\Gamma\left(1-\frac{k}{m}\right)},
$$

On the other hand, by (3.15)

$$
\frac{\partial^{m}}{\partial s^{m}} p(0, t)=t^{-(1+1 / m)} \Phi_{1 / m}^{(m)}(0)=0 .
$$

$$
\frac{\partial^{k}}{\partial s^{k}} p(s, t)=t^{-\frac{k+1}{m}} \Phi_{1 / m}^{(k)}\left(s t^{-1 / m}\right)=\frac{t^{-\frac{k+1}{m}}}{(\sqrt[m]{m})^{k}} f^{(k)}\left(\frac{s t^{-1 / m}}{\sqrt[m]{m}}\right)
$$

where $f$ is defined in (3.8) and satisfies (3.1). Therefore

$$
\frac{\partial^{k}}{\partial s^{k}} p(s, t)=\frac{t^{-\frac{k+1}{m}}}{(\sqrt[m]{m})^{k}}(-1) \frac{s t^{-1 / m}}{\sqrt[m]{m}} f\left(\frac{s t^{-1 / m}}{\sqrt[m]{m}}\right)=\frac{t^{-\frac{k+1}{m}}}{(\sqrt[m]{m})^{k+1}}(-1)^{k} s t^{-1 / m} \Phi_{1 / m}\left(s t^{-1 / m}\right)
$$


By the asymptotic expansion of the function $\Phi_{\gamma}(v)$ in case $0<\gamma<1$, (cf. [5, p.14], [11, Theorem 2.1.3] or [19]), we have for $v \geq 0$

$$
\Phi_{\gamma}(v)=Y^{\gamma-1 / 2} e^{-Y}\left\{\sum_{k=0}^{M-1} A_{k} Y^{-k}+O\left(|Y|^{-M}\right)\right\} \text { as } Y \rightarrow \infty
$$

with $Y=(1-\gamma)\left(\gamma^{\gamma} v\right)^{1 /(1-\gamma)}$, where $A_{k}$ are certain real numbers. In the particular case $\gamma=1 / m$ we have $Y_{m}=\frac{m-1}{m}\left(\frac{1}{m}\right)^{1 /(m-1)} v^{\frac{m}{m-1}}$, where $1<\frac{m}{m-1} \leq 2$. We deduce that $e^{\omega v} \Phi_{1 / m}(v) \rightarrow 0$ as $v \rightarrow \infty$ for all $\omega \in \mathbb{R}$. Hence, by (3.21) we get

$$
\lim _{s \rightarrow \infty} \frac{\partial^{m-k}}{\partial s^{m-k}} p(s, t) T^{(k-1)}(s) f=0
$$

for all $1 \leq k \leq m$. Using the above facts in (3.19), we obtain

$$
\begin{aligned}
u^{\prime}(t) & =\sum_{k=1}^{m}(-1)^{m+k} \frac{\partial^{m-k}}{\partial s^{m-k}} p(0, t) A^{k-1} f+\int_{0}^{\infty} p(s, t) A^{m} T(s) f d s \\
& =\sum_{k=1}^{m}(-1)^{m+k} \frac{t^{\frac{k-1}{m}-1}}{\Gamma\left(\frac{k-1}{m}\right)}(-1)^{m-k} A^{k-1} f+A^{m} \int_{0}^{\infty} p(s, t) T(s) f d s \\
& =\sum_{k=2}^{m} \frac{t^{\frac{k-1}{m}-1}}{\Gamma\left(\frac{k-1}{m}\right)} A^{k-1} f+A^{m} \int_{0}^{\infty} p(s, t) T(s) f d s \\
& =\sum_{k=1}^{m-1} \frac{t^{\frac{k}{m}-1}}{\Gamma\left(\frac{k}{m}\right)} A^{k} f+A^{m} u(t),
\end{aligned}
$$

proving the theorem.

Remark 3.4. In passing, we note that we have proved the following identity

$$
\frac{\partial^{m}}{\partial x^{m}} p(x, t)=(-1)^{m} \frac{\partial}{\partial t} p(x, t),
$$

which is the key in the argument of the previous theorem. Note that a similar relation has been observed in [15, Lemma 3.2 and Lemma 4.3]. Performing the Ansatz:

$$
p(x, t)=e^{i k x} e^{i \omega(k) t}
$$

where $\omega(k)$ is the dispersion relation, we obtain $\omega(k)=k^{m}$ if $m$ is odd and $\omega(k)=-k^{m}$ if $m$ is even. Hence (3.24) changes the behavior between a dispersive equation in case $m$ is odd and a diffusive equation in case $m$ is even. Note that (3.24) corresponds to the heat equation in case $m=2$ and to the homogeneous Airy equation in case $m=3$. Observe that the last is the linear part of many KdV equations.

Since

$$
u(t)=S_{1 / m}(t) f=\int_{0}^{\infty} p(s, t) T(s) f d s,
$$

solves (3.11) (and (3.12)) we note that, in terms of the temporal variable, equation (3.11) oscillate between a diffusive and dispersive behavior when the fractional exponent $1 / m$ changes between $m$ odd and $m$ even. 
Remark 3.5. We have in (3.17) established the identity

$$
\Phi_{1 / m}^{(m)}(v)=\frac{(-1)^{m+1}}{m}\left[\Phi_{1 / m}(v)+v \Phi_{1 / m}^{\prime}(v)\right] .
$$

This formula appears to be new for the Mainardi functions of rational order.

Remark 3.6. From our results and those in [10], we can deduce explicit representation of the functions $\Phi_{1 / m}(x)$, for some numbers $m \geq 3$, in terms of the improper integral

$$
\frac{1}{\pi} \int_{0}^{\infty} \cos \left(\frac{t^{k(m)}}{k(m)} \pm x t\right) d t, \quad k(m) \in \mathbb{N} .
$$

\section{REFERENCES}

[1] H. Allouba, W. Zheng, Brownian-time processes: The PDE connection and the halfderivative generator, Ann. Prob. 29 (2001), 1780-1795.

[2] H. Allouba, Brownian-time processes: The PDE connection II and the corresponding Feynman-Kac formula, Trans. Amer. Math. Soc. 354 (11) (2002), 4627-4637.

[3] W. Arendt, C. Batty, M. Hieber, F. Neubrander, Vector-valued Laplace Transforms and Cauchy Problems, Monographs in Mathematics, 96, Birkhäuser, Basel, 2001.

[4] B. Baeumer, M.M. Meerschaert, E. Nane, Brownian subordinators and fractional Cauchy problems, Trans. Amer. Math. Soc., to appear.

[5] E. Bazhlekova, Fractional Evolution Equations in Banach Spaces, Ph.D. Thesis, Eindhoven University of Technology, 2001

[6] A. S. Carasso, T. Kato, On subordinated holomorphic semigroups, Trans. Amer. Math. Soc. 327(2) (1991), 867-878.

[7] E. B. Davies, Heat Kernels and Spectral Theory, Cambridge University Press, Cambridge, 1989.

[8] D. DeBlassie, Iterated Brownian motion in an open set, Ann. Prob. 14(3) (2004), 1529-1558.

[9] K.J.-Engel, R. Nagel, One-parameter Semigroups for Linear Evolution Equations, Graduate Texts in Math.,194, Springer, New York, 2000.

[10] K. Fujii, A higher order non-linear differential equation and a generalization of the Airy function, Preprint.

[11] R. Gorenflo, Y. Luchko, F. Mainardi, Analytical properties and applications of the Wright function, Fractional Calculus and Applied Analysis, 2 (4)(1999), 383-414.

[12] V.V. Kulish, J.L. Lage, Application of fractional calculus to fluid mechanics, Journal of Fluids Engineering, 124(2002), 803-806.

[13] C. Lizama, Regularized solutions for abstract Volterra equations, J. Math. Anal. Appl. 243 (2000), 278-292.

[14] F. Mainardi, Fractional calculus: some basic problems in continuum and statistical mechanics, in: A. Carpinteri, F. Mainardi (Eds.), Fractals and Fractional Calculus in Continuum Mechanics, Springer, Wien, 1997, pp. 291-348.

[15] E. Nane, Higher order PDE's and iterated processes, Trans. Amer. Math. Soc. 360 (5) (2008), 2681-2692.

[16] P. Miller, Applied Asymptotic Analysis, Graduate Studies in Mathematics, 75, 2006.

[17] A. Pazy, Semigroups of Linear Operators and Applications to Partial Differential Equations, Applied Mathematical Sciences, Vol. 44, Springer-Verlag, 1983.

[18] J. Prüss, Evolutionary Integral Equations and Applications, Monographs Math., 87, Birkhäuser Verlag, 1993.

[19] E. M. Wright, The generalized Bessel function of order greater than one, Quarterly Journal of Mathematics (Oxford ser.) 11 (1940), 36-48.

[20] G. Zaslavsky, Fractional kinetic equation for Hamiltonian chaos. Chaotic advection, tracer dynamics and turbulent dispersion, Phys. D 76 (1994), 110-122.

University of Puerto Rico, Department of Mathematics, Faculty of Natural Sciences, Po Box 23355, PR - 00931 U.S.A.

E-mail address: keyantuo@uprrp.edu

Universidad de Santiago de Chile, Departamento de Matemática, Facultad de Ciencias, Casilla 307 Correo 2, Santiago-Chile.

E-mail address: carlos.lizama@usach.cl 